\title{
Competing zero-field Chern insulators in Superconducting Twisted Bilayer Graphene
}

\section{Dmitri Efetov ( $\nabla$ dmitri.efetov@icfo.eu )}

Institute of Photonic Sciences https://orcid.org/0000-0001-5862-0462

\section{Petr Stepanov}

ICFO - Institute of Photonic Sciences https://orcid.org/0000-0002-1121-3146

\section{Ming Xie}

University Texas Austin

\section{Takashi Taniguchi}

National Institute for Materials Science, Tsukuba, Ibaraki

\section{Kenji Watanabe}

National Institute for Materials Science https://orcid.org/0000-0003-3701-8119

\section{Xiaobo Lu}

ETH Zurich https://orcid.org/0000-0003-3149-4755

\section{Allan MacDonald}

University of Texas at Austin

\section{B. Andrei Bernevig}

Department of Physics, Princeton University

\section{Physical Sciences - Article}

Keywords: magic angle twisted bilayer graphene (MATBG), quantum electronics

Posted Date: January 13th, 2021

DOI: https://doi.org/10.21203/rs.3.rs-138250/v1

License: (c) (1) This work is licensed under a Creative Commons Attribution 4.0 International License.

Read Full License 


\section{Competing zero-field Chern insulators in Superconducting Twisted Bilayer Graphene}

Petr Stepanov $^{1 *}$, Ming Xie ${ }^{2}$, Takashi Taniguchi ${ }^{3}$, Kenji Watanabe ${ }^{3}$, Xiaobo Lu ${ }^{1}$, Allan H. MacDonald $^{2}$, B. Andrei Bernevig ${ }^{4}$ and Dmitri K. Efetov ${ }^{1 *}$

1. ICFO - Institut de Ciencies Fotoniques, The Barcelona Institute of Science and Technology, Castelldefels, Barcelona, 08860, Spain

2. Department of Physics, University of Texas at Austin, Austin, TX 78712, USA

3. National Institute of Material Sciences, 1-1 Namiki, Tsukuba, 305-0044, Japan

4. Department of Physics, Princeton University, Princeton, New Jersey 08544, USA

*Correspondence to: petr.stepanov@icfo.eu,dmitri.efetov@icfo.eu

The discovery of magic angle twisted bilayer graphene (MATBG) has unveiled a rich variety of superconducting, magnetic and topologically nontrivial phases. The existence of all these phases in one material, and their tunability, has opened new pathways for the creation of unusual gate tunable junctions. However, the required conditions for their creation - gate induced transitions between phases in zero magnetic field - have so far not been achieved. Here, we report on the first experimental demonstration of a device that is both a zero-field Chern insulator and a superconductor. The Chern insulator occurs near moiré cell filling factor $v=+1$ in a hBN non-aligned MATBG device and manifests itself via an anomalous Hall effect. The insulator has Chern number $C= \pm 1$ and a relatively high Curie temperature of $T_{c} \approx 4.5 \mathrm{~K}$. Gate tuning away from this state exposes strong superconducting phases with critical temperatures of up to $T_{c} \approx 3.5 \mathrm{~K}$. In a perpendicular magnetic field above $B>0.5 \mathrm{~T}$ we observe a transition of the $v=+1$ Chern insulator from Chern number $C= \pm 1$ to $C=3$, characterized by a quantized Hall plateau with $R_{y x}=h / 3 e^{2}$. These observations show that interaction-induced symmetry breaking in MATBG leads to zero-field ground states that include almost degenerate and closely competing Chern insulators, and that states with larger Chern numbers couple most strongly to the $B$-field. By providing the first demonstration of a system that allows gate-induced transitions between magnetic and superconducting phases, our observations mark a major milestone in the creation of a new generation of quantum electronics.

Recently discovered quantum phases in the flat-bands of $\theta_{m} \sim 1.1^{\circ}$ magic angle twisted bilayer graphene (MATBG) include correlated insulators ${ }^{1-5}(\mathrm{CI})$, superconductors ${ }^{2,6-16}$ (SC), and interaction induced correlated Chern insulators ${ }^{17-19,35-38}$ (CCI). The CCIs can occur with different Chern numbers, and have $U(4)$ valley/spin ferromagnetism in the bulk and topologically protected states at device edges. The search for the exact nature of these exotic phase $^{20-23}$ and the competition ${ }^{10,24-29}$ between them requires a complete understanding of the role of electronic interactions in the symmetry breaking of the non-interacting 4-fold spin and valley degenerate bands. The existence of multiple correlated phases in one materials platform opens up new possibilities for the creation of complex gate tunable junctions ${ }^{30,31}$. Among these the most interesting are junctions between superconducting and topological magnetic phases. The clean, gate defined homojunctions of these phases could pave new avenues for the creation of topological and spin-triplet superconductivity, as well as non-abelian particles, such as parafermions and Majorana fermions ${ }^{32}$. However, the necessary requirements for such junctions, 
namely reversible gate tuning between $\mathrm{SC}$ and CCI states in a single device in zero magnetic field, has not been previously achieved.

The occurrences of CCI phases in MATBG at integer carrier filling per moiré unit cell $v$ is a result of electronic interactions that break the system's combined inversion and timereversal symmetry $C_{2} T$. Breaking this symmetry can give rise to gapped valley polarized bands and to the formation of quantum Hall isospin ferromagnets ${ }^{33-35}$ with a well-defined correspondence $(C, v)$ between Chern numbers $C$ and electron fillings $v^{25,26}$. However, while theory predicts the existence of a variety of competing CCIs in zero magnetic field ${ }^{24,25}$, some of these states have so far only be observed at elevated perpendicular $B$-field, and hence above the critical field of the SC states. The experimental variability of the CCI state manifestation can be explained by the sensitivity of the competition between states to experimental parameters, such as strain and dielectric environment ${ }^{10}$, and also possibly by complex networks of magnetic domain walls that can obscure the quantization of the CCIs. In hexagonal boron nitride $(\mathrm{hBN})$ aligned MATBG devices with explicitly broken $C_{2}$ symmetry at the single particle level, CCIs with a quantized Hall conductance in zero magnetic field have been found at $v=+3^{18}$. However, neither SC nor CIs were observed in these devices, demonstrating that the single-particle term in the Hamiltonian that favors one sublattice over the other in aligned $\mathrm{hBN}$ devices, alters the competition between states.

Here we report on the first observation of an interaction induced anomalous Hall effect (AHE) in hBN non-aligned MATBG. The CCI occurs near $v=+1$ filling and marks the formation of two (not yet fully quantized) $C= \pm 1$ correlated Chern insulators in zero magnetic field $B=0 \mathrm{~T}$. This is the first observation of a CCI near $v=+1$ in any MATBG device, $\mathrm{hBN}$ aligned or non-aligned. The device also displays CIs with trivial gaps at $v= \pm 2$ and $v=+3$, as well as fully developed superconducting regions that allow direct gate-induced switching between SC and CCI states. Furthermore, in elevated out-of-plane $B$-field the device shows a pronounced sequence of perfectly quantized CCIs which follow the correspondence of $(C, v)$ of $( \pm 2,0),( \pm 4,0),( \pm 3, \pm 1),( \pm 2, \pm 2)$ and $( \pm 1, \pm 3)^{36-41}$. We find that these high field topological CCI states can both coexist with non-topological CI states and compete with the CCI states observed in the absence of magnetic field, in particular the $( \pm 1,1)$ and the $(3,1)$ states.

Fig. 1a shows the optical image of the four-terminal transport device, which consists of a graphite $/ \mathrm{hBN} / \mathrm{MATBG} / \mathrm{hBN}$ heterostructure with a twist angle of $\theta=1.08 \pm 0.01^{\circ}\left(n_{s}=\right.$ $\left.2.71 \times 10^{12} \mathrm{~cm}^{-2}\right)$. Crystallographic alignment between MATBG and hBN substrate layers is excluded by optical images of the naturally broken crystal edges of the individual layers, as well as by the absence of a charge neutrality gap and satellite resistance peaks in transport measurements (see SI for details). Here the longitudinal $R_{x x}=V_{x x} / I$ and Hall $R_{y x}=-V_{x y} / I$ resistance values are obtained from lock-in voltage measurements of $V_{x x}$ and $V_{x y}$, and the source-drain current $I$ (see Methods). The charge carrier density $n$ is capacitively controlled with a local back gate voltage $V_{g}$ on the graphite layer. We define the filling factor of carriers per moiré unit cell as $v=4 n / n_{s}$, where $n_{s}$ is the density of fully filled superlattice flat band and the prefactor 4 accounts for spin and valley degeneracy of each low-energy flat band.

Fig. $1 \mathrm{~b}$ (top) shows the temperature and density dependent phase diagram of the device, via a color plot of $R_{x x}$ vs. $n$ and $T$ in zero magnetic field $B$, and Fig. $1 \mathrm{~b}$ (bottom) shows the corresponding line-traces of $R_{x x}$ and $R_{y x}$ vs. $n$ at base temperature $T=30 \mathrm{mK}$. At integer filling factors of $v= \pm 2$ and $v=+3$ we observe strong, temperature activated $R_{x x}$ peaks, while $R_{y x}$ remains negligible, indicating the formation of correlated insulators with topologically trivial 
gaps, as reported in all previous studies ${ }^{2,6,9}$. In addition, the device shows dome-shaped superconducting regions in the $n-T$ phase space in which $R_{x x}=0 \Omega$ (Fig. $1 \mathrm{~b}$ and c), and which have a critical temperature of up to $T_{c} \approx 3.5 \mathrm{~K}$ (taken as $50 \%$ of normal states resistance at $v=$ -2.16). Differential resistance $d V_{x x} / d I$ measurements vs. bias current $I_{d c}$ and perpendicular magnetic field $B$ show characteristic diamond shapes (Fig. 1d), and Fraunhofer features (ED Fig. 8), with critical currents and critical magnetic field values of $I_{c}=692 \mathrm{nA}$ and $B_{c}=210 \mathrm{mT}$. These results are overall in very good agreement with values found in previous reports on superconductivity in $\mathrm{MATBG}^{2,6,9}$.

In stark contrast to all previous studies of MATBG, however, at a filling of $v=+1$, we observe a pronounced anomalous Hall effect (AHE) manifested by a non-zero Hall resistance which at $T=50 \mathrm{mK}$ reaches a value close to the quantum of conductance $R_{y x} \approx 0.9 \mathrm{~h} / \mathrm{e}^{2}$, and goes hand-in-hand with a vanishingly small longitudinal resistance that approaches $R_{x x}=0 \Omega$ (Fig. 1e). The sign of $R_{y x}$ can be flipped by applying a small perpendicular field $B<200 \mathrm{mT}$, and shows a strong hysteresis loop between up and down sweeps of the field, which are centered around $B=0 \mathrm{~T}$. The anomalous Hall effect occurs in a narrow density range from $v \approx$ +0.6 to $v \approx+1$. The strength of the hysteresis loop, which is defined as $\Delta R_{y x} / 2=\left(R_{y x}{ }^{B \downarrow}-R_{y x}{ }^{B \uparrow}\right) / 2$ $(B=0 \mathrm{~T})$, has a maximum at a filling of $v \approx+0.84\left(n=0.57 \times 10^{12} \mathrm{~cm}^{-2}\right) . \Delta R_{y x} / 2$ is quite robust at elevated temperatures, with an extracted Curie temperature of $T_{c} \approx 4.5 \mathrm{~K}$ (Fig. 1f), and follows a thermally activated dependence with an extracted energy gap $\Delta \approx 2.41 \mathrm{meV}$. Overall these findings are notably close to previous reports on magnetism in graphene moiré heterostructures ${ }^{17,18,42-44}$, which have been interpreted as manifestations of an underdeveloped correlated Chern insulator with $|C|=1$. The absence of quantization at the present time may be due to quasiparticle delocalization, possibly assisted by transport pathways along domain walls.

While at $B=0 \mathrm{~T}$ we only observe two CCI states with indices $( \pm 1,1)$, we see many more CCI states at high $B$-fields. The high $B$-field phase diagrams in Fig. 2a and b shows $R_{x x}$ and $R_{y x}$ measurements as a function of $n$ and $B$ at $T=50 \mathrm{mK}$, and Fig. 2c displays the schematic of the most dominant features of the resulting Landau Fan diagram. It consists of a multitude of regions with quantized Hall conductance $R_{y x} \sim h / C e$ and $R_{x x} \sim 0 \Omega$, which form wedge-like areas. These states follow a linear slope in the $n-B$ phase space, which is defined by the Streda formula ${ }^{45} d n / d B=C e / h$, and originate from different fillings $v$ at $B=0 \mathrm{~T}$. In addition, we also see highly resistive CIs at fillings $v= \pm 2$ and $v=+3$, which due to their topologically trivial gaps with Chern number $C=0$, form vertical regions in the $n-B$ phase space. While overall, we see a multitude of Landau level-like gaps, we find a clear hierarchy of gaps where the states with the corresponding indices of $(C, v)$ are particularly strongly pronounced - $( \pm 2,0),( \pm 4,0)$, $( \pm 3, \pm 1),( \pm 2, \pm 2)$ and $( \pm 1, \pm 3)$. These states are quantized at much lower fields and have almost an order of magnitude larger extracted gaps than typical LLs in the system, and have been recently interpreted as interaction driven CCIs, which are stabilized by a small $B$-field ${ }^{35}$.

We compare the experimental findings with the theoretical phase diagram of MATBG $^{22-25,46}$, which predicts a series of CIs and CCIs (Fig. 3). While the finer details of this phase diagram (in particular valley-spin polarizations) are not completely settled, the competition between states with different Chern numbers, if not their energetic order, is similar in most theoretical scenarios. In order to motivate the theoretical phase diagram, we consider two limits: first, the flat-band limit, where the kinetic energy of the bands is artificially tuned to zero, and second, the chiral limit, where the AA-region hopping between the MATBG bilayers is artificially tuned to zero (the AB-region coupling is still considered). In this "chiral- 
flat" limit, the system enjoys a large $U(4) \times U(4)$ symmetry, which allows for the exact determination of the ground-states of the Coulomb interaction Hamiltonian. Due to the single particle topology of MATBG, the two flat bands at each valley and spin bands can be labeled by a Chern number $C=1$ and $C=-1$, related by $C_{2}$ symmetry $^{22-24,46-49}$. In the chiral flat limit, at each filling $v$, the interaction favors the successive occupation of such Chern bands reminiscent of quantum Hall ferromagnetism, leading to many-body degenerate ground states (which can be classified by $U(4) \times U(4)$ representations) at filling $v$ with Chern number $(4-\mid v$ $\mid),(2-|v|), \ldots,(|v|-2),(|v|-4)$. We interpret these "chiral-flat" limit states as the low-energy states which compete against each other in the realistic system and which we observe in this study.

Realistic MATBG is however, not exactly in the chiral-flat limit. Perturbation away from the chiral limit (but still not considering the kinetic energy of the bands) provides insight into the nature of the correlated states: the lowest Chern number correlated states win, while the larger Chern number correlated states acquire a finite energy above the ground-state. Hence at filling $v=0, \pm 2$ the theoretical ground states have Chern number 0 , while at $v= \pm 1, \pm 3$ has theoretical ground states have Chern number $|C|=1$, all of which are now ferromagnetic in a lower $U(4)$ symmetry that still exists in the flat-band limit. A magnetic field lowers the energy of the large Chern number states with respect to those of the low Chern number states. At filling $v= \pm 1$ a first order phase transition between the low-field $|C|=1$ ground-state and the highfield $|C|=3$ ground-state is predicted to occur at $B_{2}=0.5 \mathrm{~T}^{25}$. At filling $v= \pm 2$ a first order phase transition between the low-field $|C|=0$ ground-state and the high-field $|C|=2$ groundstate is predicted to occur at $B_{1}=0.2 \mathrm{~T}$, although these numbers should only be indicative of order-of magnitude.

Perturbation theory predicts the polarization of the Chern correlated states upon introduction of kinetic energy, when the symmetry of the system lowers even further to $U(2)$ spin-charge rotation per valley. The Chern number $C=0$ and $0<|C|<4-|v|$ states at integer fillings $v$ are fully and partially intervalley coherent respectively, while the states with Chern number $|C|=4-|v|$ are valley polarized. These results are perturbative away from the chiralflat limit. The theoretical predictions are only as good as the region of validity of the perturbation allows. Numerical results based on exact diagonalization and $\mathrm{DMRG}^{23,46,50}$ suggest that some of the Chern insulating ground-states, particularly at $v= \pm 3$, do not survive for realistic MATBG parameters, and that a competition occurs between (nematic) metal, momentum $M(\pi)$ stripe, and $K$-CDW orders and metallic states with no broken symmetries.

We now analyze the correspondence between the experimental and the theoretical phase diagrams. At $v= \pm 2$, the experiment and theory are in agreement with a zero field Chern number $C=0$ state and an in-field $|C|=2$ state. In zero field at $v=+3$, the experimental Chern number is $C=0$, while for $v=-3$ we do not find any experimental signatures of neither, the CI nor a CCI. These zero field states conflict with the perturbation-derived $|C|=1$ states, however at high $B$-field these transition to $|C|=1$ states, as predicted by theory. We find that the theoretically predicted low field $|C|=1$ state is the only possible option arising from a translationally invariant interaction driven Chern bands at at $v= \pm 3$, although $C=0$ insulators are allowed if mixing with remote bands plays a role ${ }^{24}$. Its absence could suggests the presence of translational symmetry-broken states at this filling, which is further supported by numerical results $^{25}$. At $v=+1$, the experimental discovery, presented here of a $|C|=1$ is in tune with the theoretically predicted ground-state. Most strikingly, the in-field transition from the $|C|=1$ to 
the higher, $|C|=3$ state also corresponds to the theoretically predicted state. Such agreement at $v=+1$ suggests that further samples can reveal similar physics at $v=-1$.

We examine the observed $B$-field induced transitions of the phases originating from the $v=+1$ filling in detail in Fig. $4 \mathrm{~b}$, which shows a color map of $R_{y x}$ vs. $n$ and $B$. At low fields $B$ $<0.5 \mathrm{~T}$ the phase diagram is mainly defined by the AHE hysteresis loop on the hole doped side of $v<+1$. The center of the hysteresis loop shifts in $B$-field in agreement with the Streda formula for a Chern number of $C=-1$ for positive and $C=+1$ for negative $B$-field (Fig. 4a). The sign of the Chern numbers is consistent with the sign of $R_{y x}$ for the AHE, which maintains positive values in positive $B$, while the normal Hall effect at higher $n$ produces a negative $R_{y x}$. While $\Delta R_{y x}$ is strongest around $v \approx+0.84$, close to $v=+1$ it is strongly suppressed and its coercive $B$-field values are increased (Fig. $4 \mathrm{~d}$ and ED Fig. 4). Sharply at $v>+1$ we observe a sign reversal of $\Delta R_{y x}$, which however becomes much weaker. Changes in the sense of the hysteresis loop and the magnitude of the hysteresis loop across the gap are expected to be a common feature of Chern insulators ${ }^{43,51}$ (See SI and Fig. 4e). These low field CCI states gradually disappear above $B>0.5 \mathrm{~T}$, and we observe the onset of an CCI which also follows the Streda formula with Chern number of $C=+3$ and shows well quantized Hall plateaus $R_{y x}=$ $h / 3 e^{2}\left(R_{x x}=0 \Omega\right)$ shown in Fig. 4c. The absence of a $C=-3$ state is likely due to competing metallic states with no broken symmetries as explained in the supplementary material. These findings clearly establish that just like theory predicts, the ground states of MATBG consist of closely competing CCIs, for which $B$-field can act as a tuning knob, which couples strongest to the states with higher Chern number.

To summarize - our findings shed new light on the underlying ground states of MATBG, and show that even in zero-field these form nearly degenerate and competing interaction-induced Chern insulators, which can be further tuned by weak magnetic fields. The exact sequence of these phases in zero-field and their evolution in $B$-field gives detailed information about the competition of these phases and allows to understand the exact microscopic mechanism that drive their formation, through comparisons to ongoing theoretical models. The zero-field coexistence and gate tunability of these magnetically and topological nontrivial phases with superconducting phases, presents a remarkable opportunity to electronically hybridize these phases through engineering of complex gate induced junctions, and will lead to the creation of ever more complex quantum phases based on the MATBG platform. 


\section{References:}

1. Cao, Y. et al. Correlated insulator behaviour at half-filling in magic-angle graphene superlattices. Nature 556, 80-84 (2018).

2. Lu, X. et al. Superconductors, Orbital Magnets, and Correlated States in Magic Angle Bilayer Graphene. Nature 574, 653-657 (2019).

3. Wong, D. et al. Cascade of electronic transitions in magic-angle twisted bilayer graphene. Nature 582, 198-202 (2020).

4. Zondiner, U. et al. Cascade of phase transitions and Dirac revivals in magic-angle graphene. Nature 582, 203-208 (2020).

5. Po, H. C., Zou, L., Vishwanath, A. \& Senthil, T. Origin of Mott Insulating Behavior and Superconductivity in Twisted Bilayer Graphene. Phys. Rev. X 8, 31089 (2018).

6. Cao, Y. et al. Unconventional superconductivity in magic-angle graphene superlattices. Nature 556, 43-50 (2018).

7. Dodaro, J. F., Kivelson, S. A., Schattner, Y., Sun, X. Q. \& Wang, C. Phases of a phenomenological model of twisted bilayer graphene. Phys. Rev. B 98, 075154 (2018).

8. Xie, F., Song, Z., Lian, B. \& Bernevig, B. A. Topology-Bounded Superfluid Weight in Twisted Bilayer Graphene. Phys. Rev. Lett. 124, 167002 (2020).

9. Yankowitz, M. et al. Tuning superconductivity in twisted bilayer graphene. Science 363, 1059-1064 (2019).

10. Stepanov, P. et al. Untying the insulating and superconducting orders in magic-angle graphene. Nature 583, 375-378 (2020).

11. Balents, L., Dean, C. R., Efetov, D. K. \& Young, A. F. Superconductivity and strong correlations in moiré flat bands. Nat. Phys. 16, 725-733 (2020).

12. Wu, F., Macdonald, A. H. \& Martin, I. Theory of Phonon-Mediated Superconductivity in Twisted Bilayer Graphene. Phys. Rev. Lett. 121, (2018).

13. Lian, B., Wang, Z. \& Bernevig, B. A. Twisted Bilayer Graphene: A Phonon-Driven Superconductor. Phys. Rev. Lett. 122, 1-26 (2019).

14. González, J. \& Stauber, T. Kohn-Luttinger Superconductivity in Twisted Bilayer Graphene. Phys. Rev. Lett. 122, 26801 (2019).

15. Isobe, H., Yuan, N. F. Q. \& Fu, L. Unconventional Superconductivity and Density Waves in Twisted Bilayer Graphene. Phys. Rev. X 8, 41041 (2018).

16. Liu, C.-C., Zhang, L.-D., Chen, W.-Q. \& Yang, F. Chiral Spin Density Wave and $\$ \mathrm{~d}+\mathrm{id} \$$ Superconductivity in the Magic-Angle-Twisted Bilayer Graphene. Phys. Rev. Lett. 121, 217001 (2018).

17. Sharpe, A. L. et al. Emergent ferromagnetism near three-quarters filling in twisted bilayer graphene. Science 365, 605-608 (2019).

18. Serlin, M. et al. Intrinsic quantized anomalous Hall effect in a moiré heterostructure. Science 367, 900 (2020).

19. Jiang, Y. et al. Charge order and broken rotational symmetry in magic-angle twisted bilayer graphene. Nature 573, 91-95 (2019).

20. Bistritzer, R. \& Macdonald, A. H. Moiré bands in twisted double-layer graphene. PNAS 108, 12233-12237 (2011).

21. Koshino, M. et al. Maximally Localized Wannier Orbitals and the Extended Hubbard Model for Twisted Bilayer Graphene. Phys. Rev. X 8, (2018).

22. Kang, J. \& Vafek, O. Symmetry, Maximally Localized Wannier States, and a LowEnergy Model for Twisted Bilayer Graphene Narrow Bands. Phys. Rev. X 8, 31088 (2018).

23. Kang, J. \& Vafek, O. Strong Coupling Phases of Partially Filled Twisted Bilayer Graphene Narrow Bands. Phys. Rev. Lett. 122, 246401 (2019).

24. Xie, M. \& MacDonald, A. H. Nature of the Correlated Insulator States in Twisted 
Bilayer Graphene. Phys. Rev. Lett. 124, 97601 (2020).

25. Lian, B. et al. TBG IV: Exact Insulator Ground States and Phase Diagram of Twisted Bilayer Graphene. arXiv Prepr. arXiv2009.13530 (2020).

26. Xie, M. \& MacDonald, A. H. Weak-field Hall resistivity and spin/valley flavor symmetry breaking in MAtBG. arXiv Prepr. arXiv2010.07928 (2020).

27. Saito, Y., Ge, J., Watanabe, K., Taniguchi, T. \& Young, A. F. Independent superconductors and correlated insulators in twisted bilayer graphene. Nat. Phys. 16, 926-930 (2020).

28. Arora, H. S. et al. Superconductivity in metallic twisted bilayer graphene stabilized by WSe2. Nature 583, 379-384 (2020).

29. Liu, X. et al. Tuning electron correlation in magic-angle twisted bilayer graphene using Coulomb screening. arXiv Prepr. arXiv2003.11072 (2020).

30. de Vries, F. K. et al. Gate-Defined Josephson Junctions in Magic-Angle Twisted Bilayer Graphene. arXiv Prepr. arXiv2011.00011 (2020).

31. Rodan-Legrain, D. et al. Highly Tunable Junctions and Nonlocal Josephson Effect in Magic Angle Graphene Tunneling Devices. arXiv Prepr. arXiv2011.02500 (2020).

32. Lian, B., Sun, X.-Q., Vaezi, A., Qi, X.-L. \& Zhang, S.-C. Topological quantum computation based on chiral Majorana fermions. Proc. Natl. Acad. Sci. 115, 10938 (2018).

33. Nomura, K. \& MacDonald, A. H. Quantum Hall Ferromagnetism in Graphene. Phys. Rev. Lett. 96, 256602 (2006).

34. Song, Y. J. et al. High-resolution tunnelling spectroscopy of a graphene quartet. Nature 467, 185-189 (2010).

35. Young, A. F. et al. Spin and valley quantum Hall ferromagnetism in graphene. Nat. Phys. 8, 550-556 (2012).

36. Das, I. et al. Symmetry broken Chern insulators and magic series of Rashba-like Landau level crossings in magic angle bilayer graphene. arXiv Prepr. arXiv2007.13390 (2020).

37. Nuckolls, K. P. et al. Strongly Correlated Chern Insulators in Magic-Angle Twisted Bilayer Graphene. arXiv Prepr. arXiv2007.03810 (2020).

38. Wu, S., Zhang, Z., Watanabe, K., Taniguchi, T. \& Andrei, E. Y. Chern Insulators and Topological Flat-bands in Magic-angle Twisted Bilayer Graphene. arXiv Prepr. arXiv2007.03735 (2020).

39. Saito, Y. et al. Hofstadter subband ferromagnetism and symmetry broken Chern insulators in twisted bilayer graphene. arXiv Prepr. arXiv2007.06115 (2020).

40. Saito, Y. et al. Isospin Pomeranchuk effect and the entropy of collective excitations in twisted bilayer graphene. arXiv Prepr. arXiv2008.10830 (2020).

41. Choi, Y. et al. Tracing out Correlated Chern Insulators in Magic Angle Twisted Bilayer Graphene. arXiv Prepr. arXiv2008.11746 (2020).

42. Chen, G. et al. Tunable correlated Chern insulator and ferromagnetism in a moiré superlattice. Nature 579, 56-61 (2020).

43. Polshyn, H. et al. Electrical switching of magnetic order in an orbital Chern insulator. Nature (2020) doi:10.1038/s41586-020-2963-8.

44. Chen, S. et al. Electrically tunable correlated and topological states in twisted monolayer-bilayer graphene. arXiv Prepr. arXiv2004.11340 (2020).

45. Streda, P. Theory of quantised Hall conductivity in two dimensions. J. Phys. C Solid State Phys. 15, L717-L721 (1982).

46. Bultinck, N. et al. Ground State and Hidden Symmetry of Magic-Angle Graphene at Even Integer Filling. Phys. Rev. X 10, 31034 (2020).

47. Song, Z. et al. All Magic Angles in Twisted Bilayer Graphene are Topological. Phys. 
Rev. Lett. 123, 36401 (2019).

48. Po, H. C., Zou, L., Senthil, T. \& Vishwanath, A. Faithful tight-binding models and fragile topology of magic-angle bilayer graphene. Phys. Rev. B 99, 195455 (2019).

49. Liu, J. \& Dai, X. Theories for the correlated insulating states and quantum anomalous Hall phenomena in twisted bilayer graphene. (2019).

50. Xie, F. et al. TBG VI: An Exact Diagonalization Study of Twisted Bilayer Graphene at Non-Zero Integer Fillings. arXiv Prepr. arXiv2010.00588 (2020).

51. Bernevig, B. A. et al. TBG V: Exact Analytic Many-Body Excitations In Twisted Bilayer Graphene Coulomb Hamiltonians: Charge Gap, Goldstone Modes and Absence of Cooper Pairing. arXiv Prepr. arXiv2009.14200 (2020).

52. Zhu, J., Su, J.-J. \& MacDonald, A. H. Voltage-Controlled Magnetic Reversal in Orbital Chern Insulators. Phys. Rev. Lett. 125, 227702 (2020).

Acknowledgements:

We are grateful for fruitful discussions with Frank H. L. Koppens and Ashvin Vishwanath. D.K.E. acknowledges support from the Ministry of Economy and Competitiveness of Spain through the "Severo Ochoa" program for Centres of Excellence in R\&D (SE5-0522), Fundació Privada Cellex, Fundació Privada Mir-Puig, the Generalitat de Catalunya through the CERCA program, funding from the European Research Council (ERC) under the European Union's Horizon 2020 research and innovation programme (grant agreement No. 852927)" and the La Caixa Foundation. B.A.B. was supported by the DOE Grant No. DE-SC0016239, the Schmidt Fund for Innovative Research, Simons Investigator Grant No. 404513, the Packard Foundation, the Gordon and Betty Moore Foundation through Grant No. GBMF8685 towards the Princeton theory program, and a Guggenheim Fellowship from the John Simon Guggenheim Memorial Foundation. Further support was provided by the NSF-EAGER No. DMR 1643312, NSFMRSEC No. DMR-1420541 and DMR-2011750, ONR No. N00014-20-1-2303, Gordon and Betty Moore Foundation through Grant GBMF8685 towards the Princeton theory program, BSF Israel US foundation No. 2018226, and the Princeton Global Network Funds. P.S. acknowledges support from the European Union's Horizon 2020 research and innovation programme under the Marie Skłodowska-Curie grant agreement No. 754510. A.H.M and M.X were supported by DOE grant DE- FG02-02ER45958 and Welch Foundation grant F1473.

Author contributions:

D.K.E and P.S. conceived and designed the experiments; P.S. fabricated the devices and performed the measurements; P.S., D.K.E., B. A. B., M.X. and A.H.M. analyzed the data; B.A.B, M.X. and A.H.M performed the theoretical modeling; T.T. and K.W. contributed materials; D.K.E. and X. L. supported the experiments: P.S., D.K.E., B. A. B, M.X. and A.H.M wrote the paper.

Supplementary Information is available for this paper.

Correspondence and requests for materials should be addressed to P.S. and D.K.E.

Competing interest. Authors claim no competing interest.

\section{Methods and materials.}

Device fabrication.

Our devices are fabricated using a "cut-and-stack" dry transfer method (ED Fig. 1). We assemble our heterostructure from top to bottom using a sacrificial poly-bis-phenol A carbonate (PC) layer placed on top of a PDMS stamp (polydimethylsiloxane). All flakes used for the 
assembly are prepared by mechanical exfoliation process on $\mathrm{Si}^{++} / \mathrm{SiO}_{2}$ chips. First, we pick up a top hBN flake, followed by a subsequent pick up of the first graphene half, which was precut in two pieces using an atomic force microscopy tip. During the next step, the transfer stage is rotated by $\sim 1.1^{\circ}$ introducing an interlayer twist between graphene layers, followed by a second graphene pickup process. The partial hBN/MATBG stack is then used to pick up a bottom $\mathrm{hBN}$ flake $(\sim 7 \mathrm{~nm})$, followed by a pick-up of a graphite stripe $(\sim 3 \mathrm{~nm}$ thick) that is used as a highquality local back gate. At the next steps, the quadruple stack hBN/MATBG/hBN/graphite is etched into a multi-terminal Hall bar geometry and coupled to 1D metallic contacts $(\mathrm{Cr} / \mathrm{Au}$ $5 / 40 \mathrm{~nm})$.

\section{Measurements.}

Transport measurement were performed in a cryogen-free dilution refrigerator using lock-in amplifiers with low $\mathrm{AC}$ excitation current in the range 1-5 $\mathrm{nA}$ at the excitation frequency of 19.111 Hz. The direct current measurements shown in ED Fig. 9 were performed using a SR560 DC voltage preamplifier in combination with a Keithley 2700 multimeter. Most of the data shown in this study is taken at base temperatures of the dilution refrigerator $30-50 \mathrm{mK}$ and available magnetic fields up to $8 \mathrm{~T}$.

Twist angle extraction.

To extract the twist angle we analyze the phase diagram shown on the ED Fig. 3. Using the relation $n_{s}=8 \theta^{2} / \sqrt{3} a^{2}$, where $\theta$ is the interlayer twist angle, $n_{s}$ is the charge carrier density corresponding to the fully filled superlattice unit cell and $a=0.246 \mathrm{~nm}$ is the interatom distance in single layer graphene, we determine the twist angle in our device $\theta=1.08 \pm 0.01^{\circ} . n_{s}$ is derived from the quantum oscillations emanating outside of the fully filled flat bands. 
Figures.

a

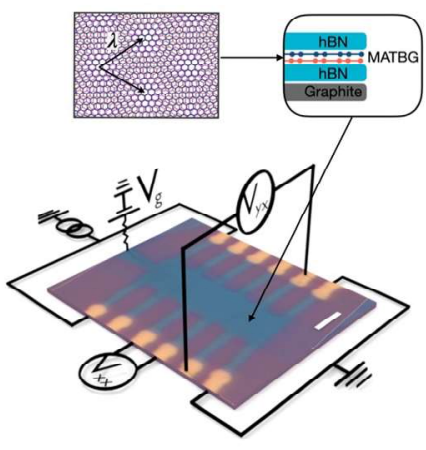

b

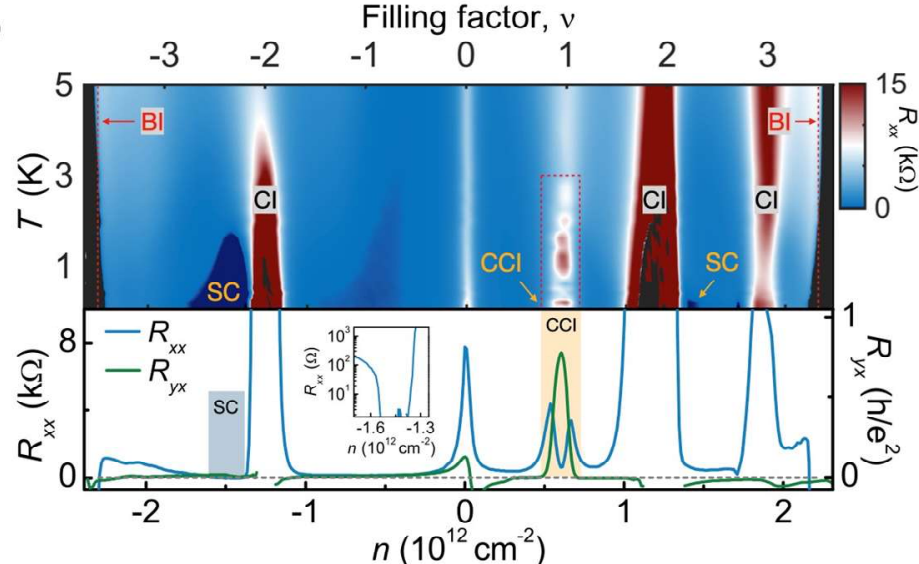

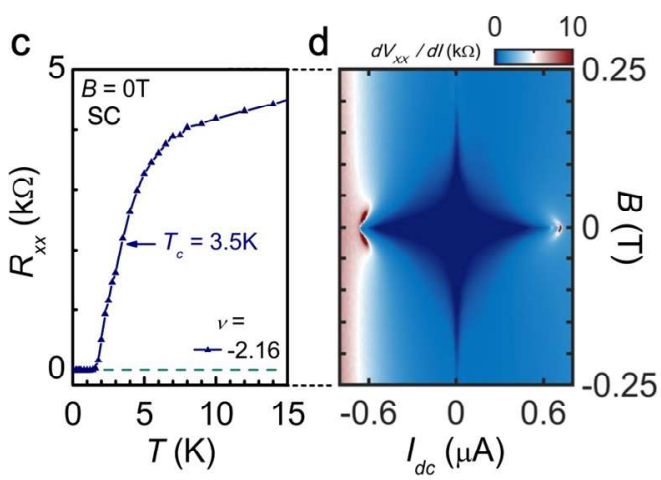

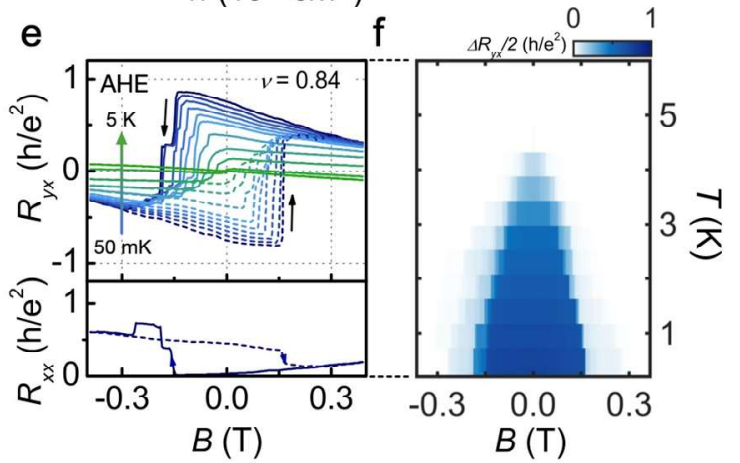

Figure 1. | Coexistence of magnetism and superconductivity in MATBG. (a) Optical image and experimental transport measurements setup of the locally gated MATBG device. (b) Upper panel. Colormap $R_{x x}$ vs. $n$ and $T$. A multitude of emergent strongly correlated phases including $\mathrm{SCs}, \mathrm{CIs}$ and CCI states coexisting in the same phase space. Dark blue regions indicate SC phases with $R_{x x}$ values below the noise level. Black regions indicate data points with quenched drain current. BI denotes band insulators. Lower panel shows $R_{x x}$ and $R_{y x}$ vs. $n$ line traces at 20 $\mathrm{mT}$. The data points, for which drain current quenches, were dropped out. The sample is tuned by the back gate between AHE and SC phases. The inset demonstrates a zoom-in image around the SC pocket with vanishing longitudinal resistance values. (c) Temperature dependence $R_{x x}$ vs. $T$ of the superconducting domain at optimal doping $v=-2.16$ shows critical temperature $T_{c}$ $=3.5 \mathrm{~K}$ (defined by the temperature of $50 \%$ of the normal metal state resistance). (d) Differential resistance $d V_{x x} / d I$ vs. direct current bias $I_{d c}$ and magnetic field $B$. A "diamond" like feature corresponds to SC phase with optimal doping at $v=-2.16$. (e) Upper panel. Hall resistance $R_{y x}$ vs. $B$ taken for increasing and decreasing magnetic fields. Hysteresis loops are indicative of the incipient Chern insulator $|C|=1$ at filling $v=+0.84$. Black arrows show the $B$ field sweep directions. Lower panel shows $R_{x x}$ vs. $B$ line trace taken at $T=50 \mathrm{mK}$. (f) AHE resistance $\Delta R_{y x} / 2$ vs. $B$ and $T$ taken at $v=+0.84 . \Delta R_{y x} / 2$ is defined by subtracting $R_{y x}(B)$ as magnetic field decreases from $R_{y x}(B)$ as $B$ increases $\Delta R_{y x} / 2=\left(R_{y x} B \downarrow-R_{y x} B \uparrow\right) / 2$. The hysteresis disappears above $T_{C} \sim 4.5 \mathrm{~K}$. 


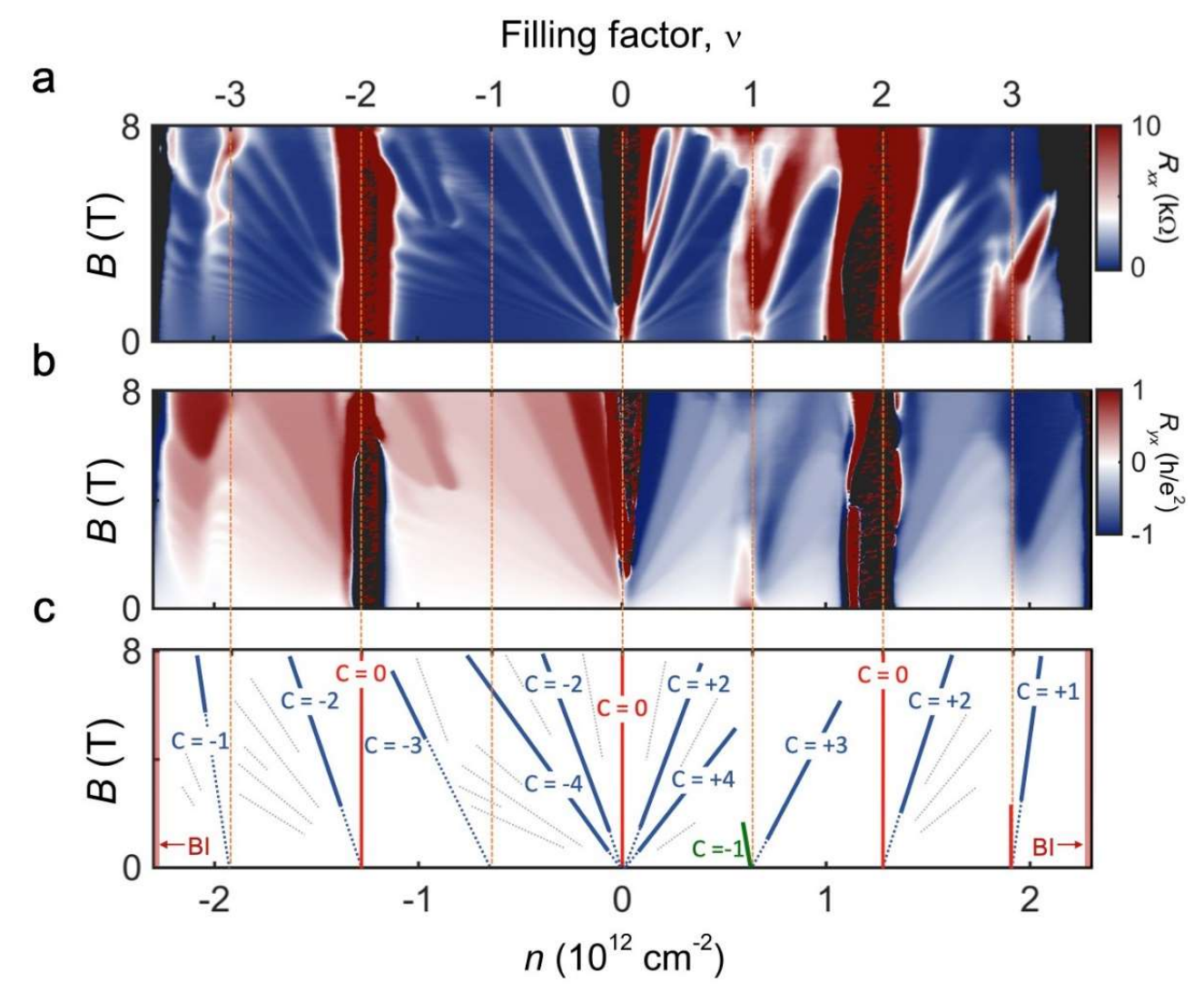

Figure 2. $\mid$ Chern insulators in MATBG at T=50 $\mathbf{~ m K}$. (a)-(b) $R_{x x}$ and $R_{y x}$ vs $n$ and $B$ over the full range of charge carrier densities of the low-energy flat bands. The phase space exhibits a zero-field Chern number -1 insulator (green solid line) and a set of high-field Chern insulators carrying numbers $\pm 1, \pm 2$, and \pm 3 (solid blue lines). We observe the full set of Chern insulators emanating from partial fillings of the superlattice unit cell $(v, C)=( \pm 1, \pm 3),( \pm 2, \pm 2),( \pm 3, \pm 1)$. (c) Schematic image, illustrating the experimental phase diagram shown in (a)-(b). The solid lines correspond to well developed Chern insulators with Hall conductance quantized at integer number of $e^{2} / h$ in the broad regions of the phase space in (b). Dashed lines show other quantum Hall states. 


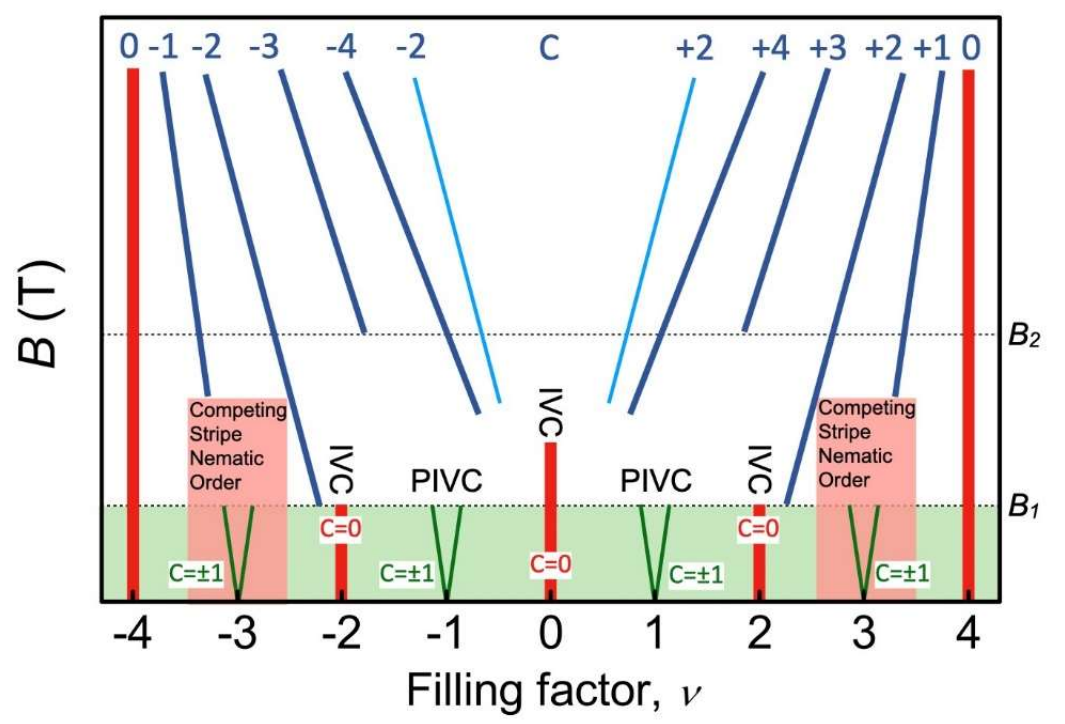

Figure 3. Phase diagram of MATBG in a magnetic field. Large magnetic field favors the largest Chern number possible many-body states given the single-particle MATBG bands. These states exhibit the maximum valley polarization allowed by the filling. At low fields, the system undergoes phase transitions from the high-field ground-states, and the theoretical situation is more complex. While at filling 0 and \pm 2 , the many-body states are correlated insulators with zero Chern number and exhibit inter-valley coherence, at filling \pm 3 , there is strong competition between a correlated $C= \pm 1$ Chern insulator and several $C=0$ translational or rotational symmetry broken states. Experimentally, no CCI is obtained at this filling, indicating that the $\mathrm{CDW}$ or nematic states win. At filling \pm 2 , the ground-state switches from $|C|=2$ in high field to $C=0$ at low field, in agreement with experiment. Nontrivially, at filling \pm 1 , the ground-state switches from $|C|=3$ in high field to $|C|=1$ in low field, in agreement with our new experimental results. Dark blue lines above $B_{1}$ show valley polarized CCI states. PIVC denotes partially inter-valley coherent and IVC - inter-valley coherent states. 

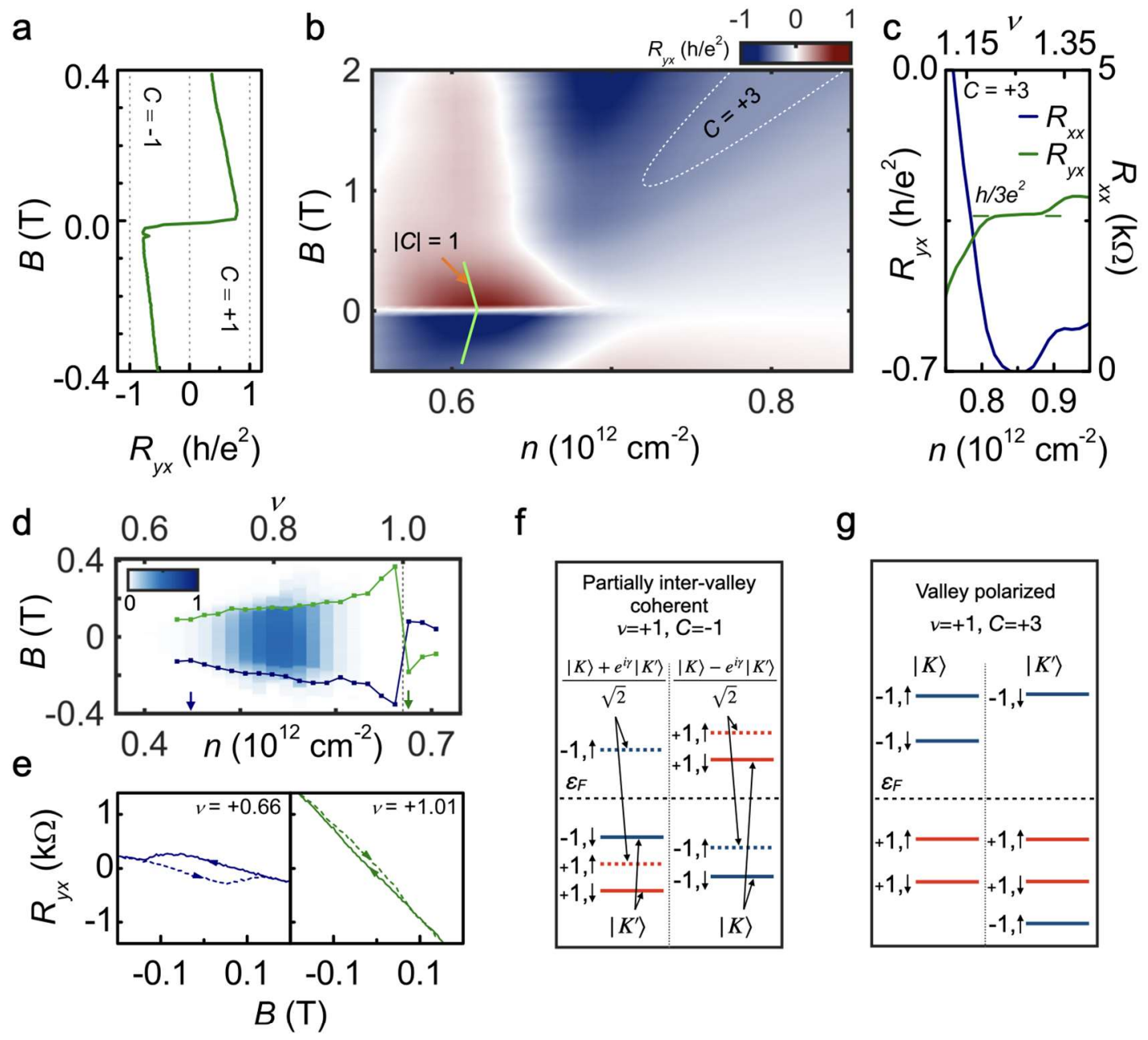

g

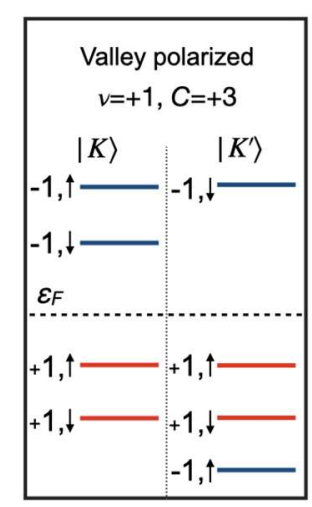

Figure 4. | Competing zero-field Chern insulators in MATBG at $\boldsymbol{T}=\mathbf{5 0} \mathbf{~ m K}$. (a) $R_{y x}$ vs. $B$ trace taken along the green line shown in (b). The slope of $R_{y x}$ minimizes when the line is chosen along $d n / d B= \pm e / h$. (b) $R_{y x}$ vs. $n$ and $B$ close to $v=+1$. The phase space exhibits zerofield Chern number $1 \mathrm{CCI}$ (green line) and high-field Chern number $3 \mathrm{CCI}$ (outlined by the white dashed line). (c) $R_{y x}$ and $R_{x x}$ vs $n$ taken at $B=2.5 \mathrm{~T}$ exhibits a single quantum Hall-like fully quantized $h / 3 e^{2}$ plateau indicative of a CCI with Chern number 3. (d) AHE resistance $\Delta R_{y x} / 2$ vs. $n$ and $B$ taken from the pair of contacts adjacent to one in Fig. 1e. Green and blue symbols show coercive field values. Note the switch of the magnetization sign upon crossing $v=+1$ (dashed line). Colorscale is set to von Klitzing constant $h / e^{2}$. (e) $R_{y x}$ vs. $B$ line traces taken at charge carrier densities color-coded to arrows shown in (d). (f)-(g) Schematics of the $C=-1$ and $C=+3$ states at filling $v=+1$. Per graphene valley, the two bands of MATBG can be transformed into two bands one with Chern number 1 , the other with Chern number -1 . The ground-states of the interacting Hamiltonian (exact in some limit) then correspond to specific ways of filling these states. For $C=-1$, we find that a partially valley coherent state (dashed lines, basis formed by wavefunctions from the two valleys) is preferred, while for $C=+3$ (the high-field ground-state), the largest valley polarization state wins. 
Figures

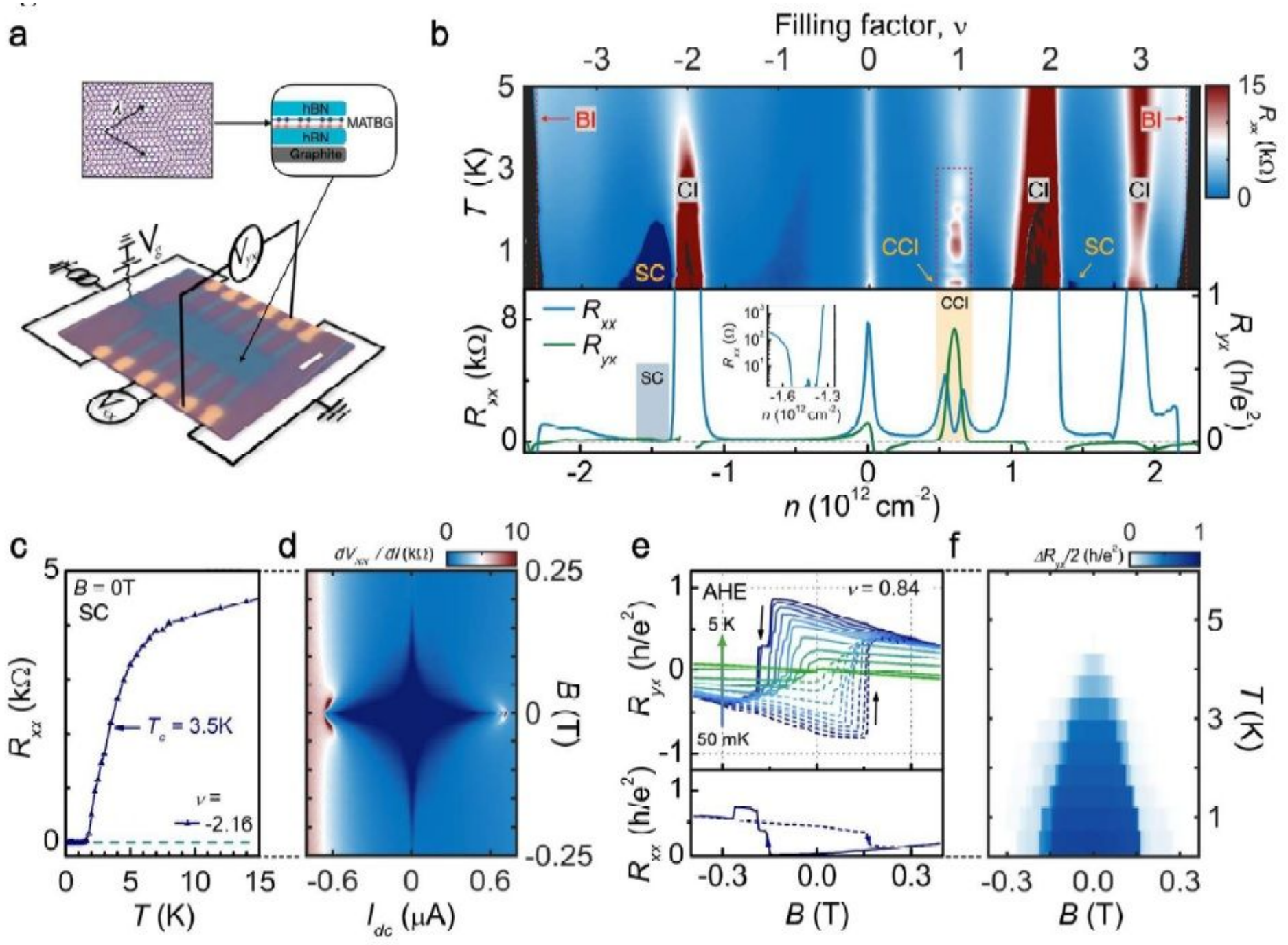

Figure 1

Coexistence of magnetism and superconductivity in MATBG. (See Manuscript file for full caption) 
Filling factor, $v$

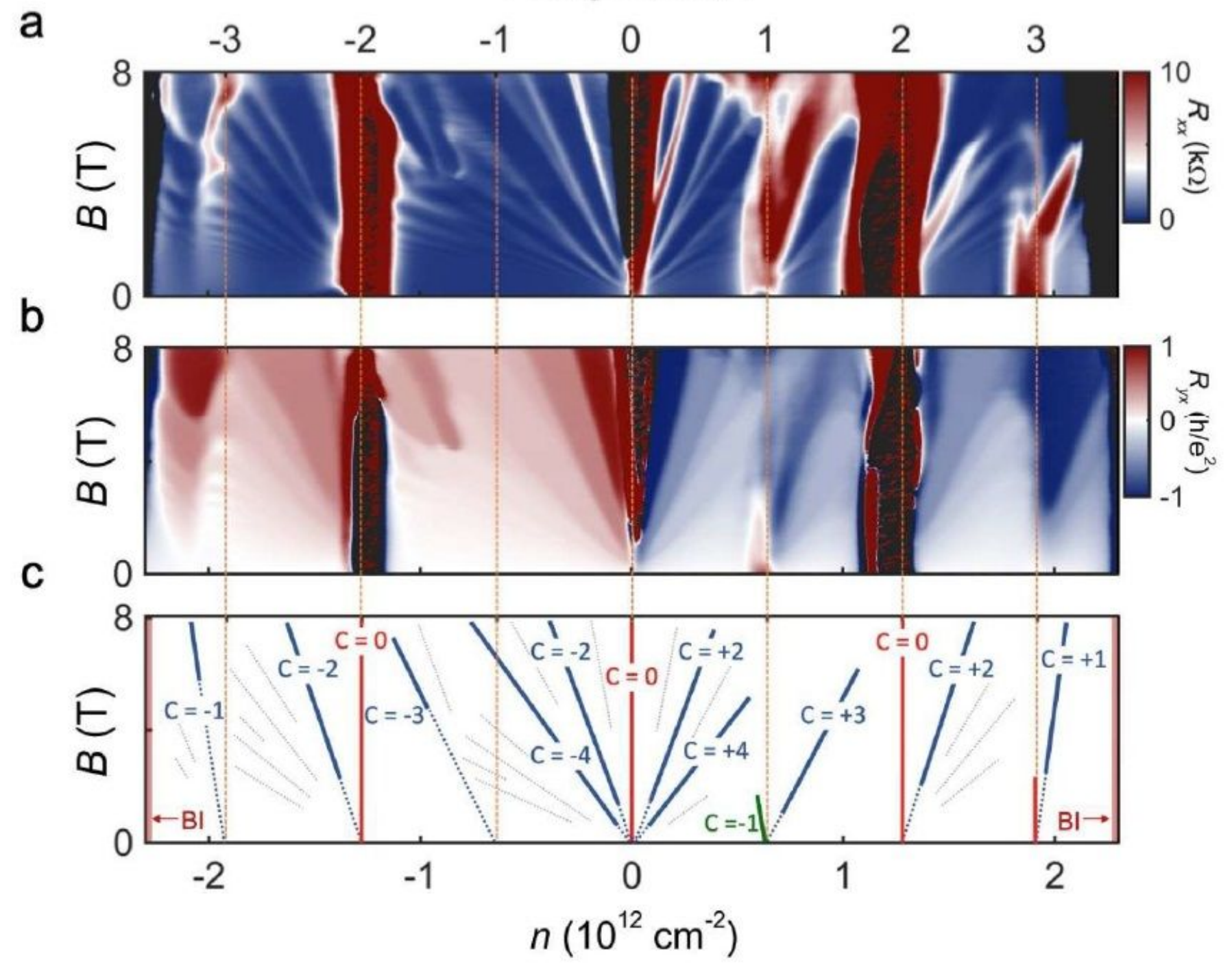

Figure 2

Chern insulators in MATBG at T=50 mK. (a)-(b) (See Manuscript file for full caption) 


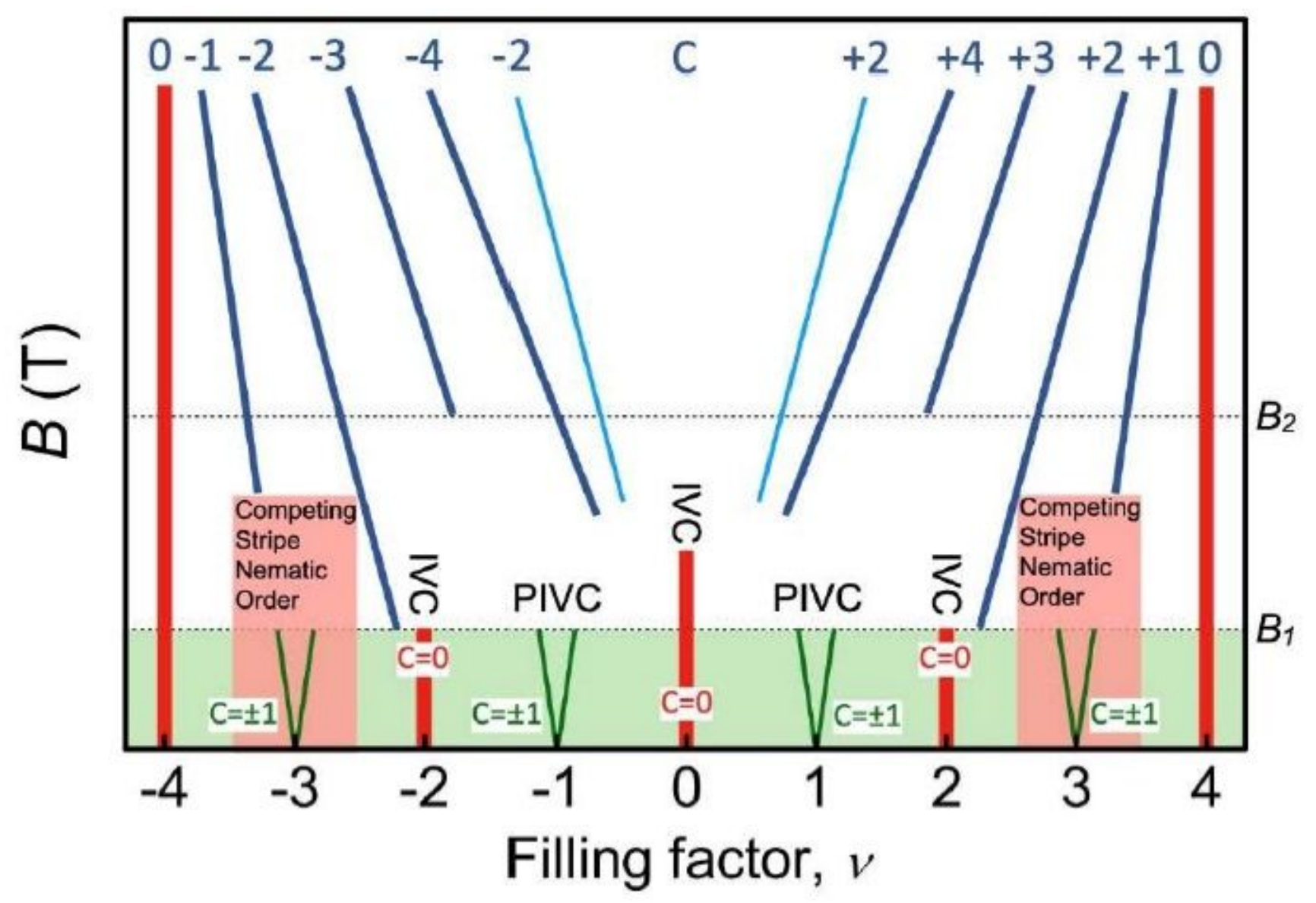

Figure 3

Phase diagram of MATBG in a magnetic field. (See Manuscript file for full caption) 


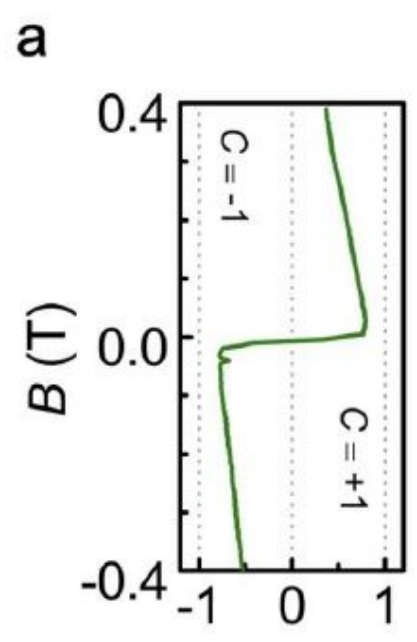

$$
R_{y x}\left(\mathrm{~h} / \mathrm{e}^{2}\right)
$$
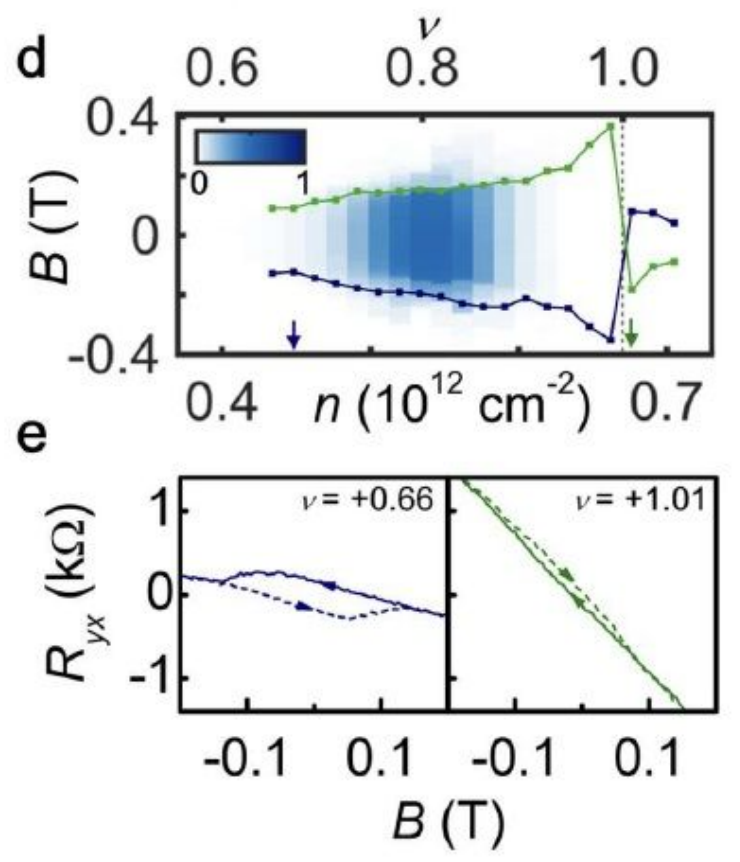

b

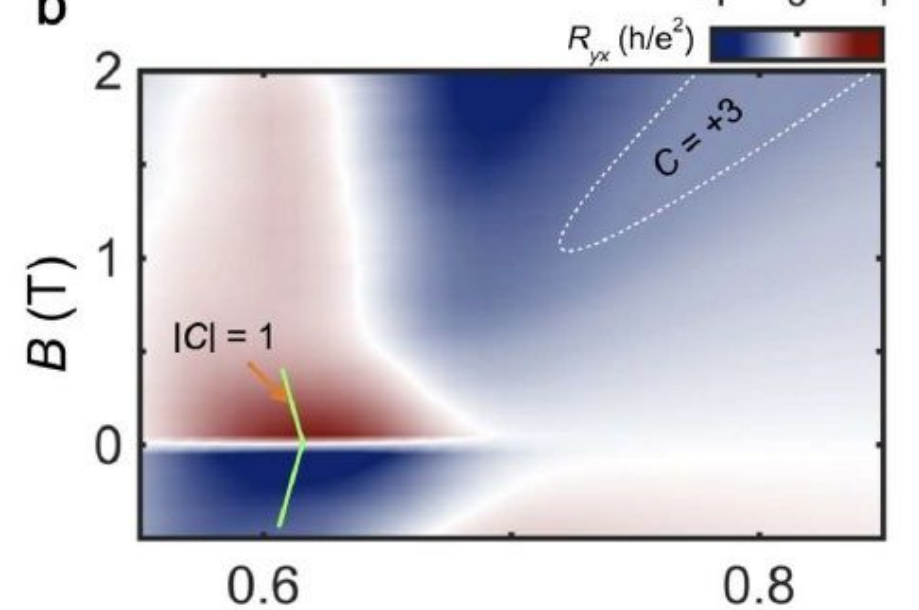

$n\left(10^{12} \mathrm{~cm}^{-2}\right)$

$f$

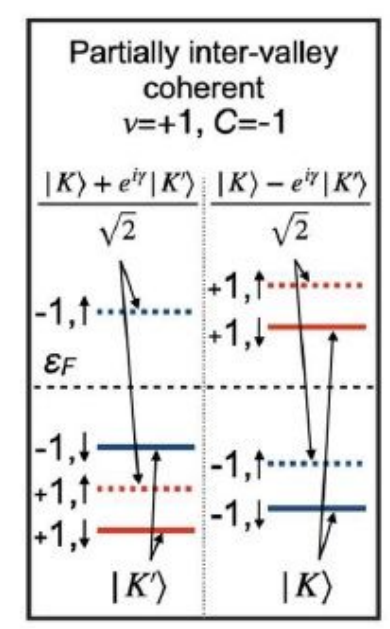

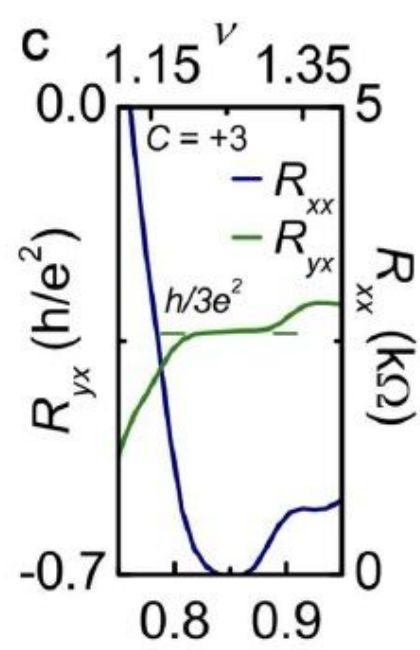

$n\left(10^{12} \mathrm{~cm}^{-2}\right)$ g

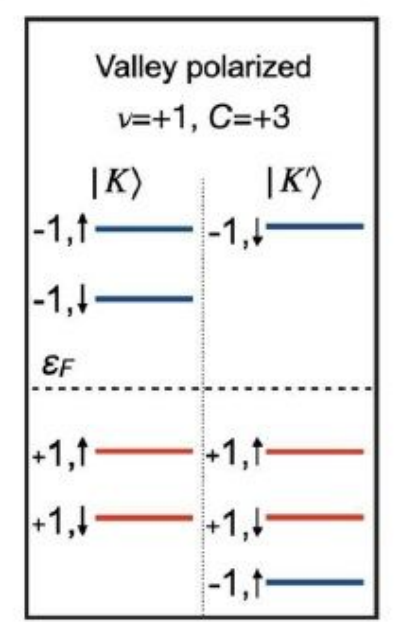

Figure 4

Competing zero-field Chern insulators in MATBG at T=50 mK.(See Manuscript file for full caption)

\section{Supplementary Files}

This is a list of supplementary files associated with this preprint. Click to download.

- SI.pdf 